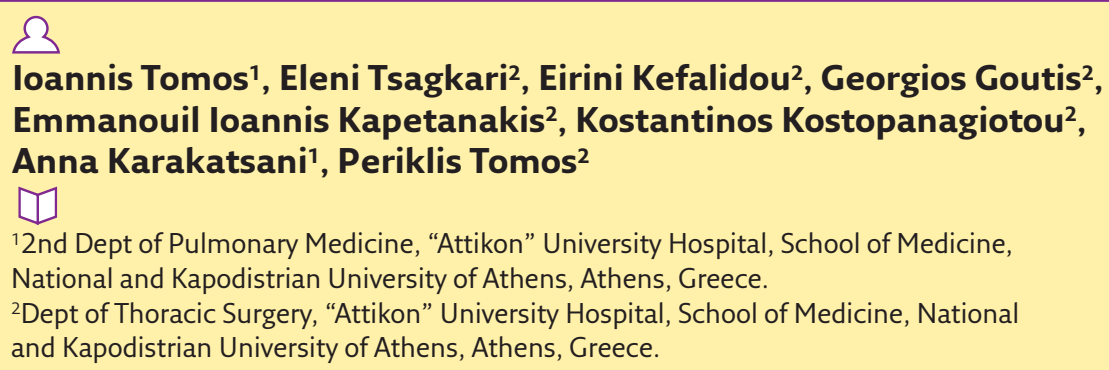

\title{
Bilateral nodular opacities and hilar node enlargement in a 73-year-old man with cough
}

\section{Case report}

A 73-year-old Caucasian male, ex-smoker with a smoking history of 50 pack-years, presented to our department complaining of chronic cough, gradually progressive shortness of breath on exertion and fatigue during recent months. He did not report any chest pain or fever. The patient's medical history only included arterial hypertension, while in the past he worked in the stone crushing industry.

On examination, the patient was afebrile, with a heart rate of 75 beats. $\mathrm{min}^{-1}$, blood pressure of $120 / 80 \mathrm{mmHg}$, a respiratory rate of 15 breaths. $\mathrm{min}^{-1}$, and an oxygen saturation of $93 \%$ on room air. He was alert and oriented. Physical examination revealed normal auscultation and percussion, with no clubbing, cyanosis, or oedema. The rest of his physical examination was unremarkable.

Laboratory examination findings were normal. Specifically, his haemoglobin level was $13 \mathrm{~g} \cdot \mathrm{dL}^{-1}$, white blood cells count was 9310 per $\mu \mathrm{L}$, and platelets were 431000 per $\mu \mathrm{L}$. Serum sodium level was $136 \mathrm{mmol} \cdot \mathrm{L}^{-1}$, potassium was $4.2 \mathrm{mmol} \cdot \mathrm{L}^{-1}$ and creatinine was $0.8 \mathrm{mg} \cdot \mathrm{dL}^{-1}$. D-dimer was also normal. The patient's ECG demonstrated normal sinus rhythm. A chest radiograph showed diffuse, small rounded opacities (figure 1), predominantly in the upper lobes of the lung and a bilateral enlargement of the hilar lymph nodes.

\section{Task 1 \\ What would your next investigations for the patient be?}

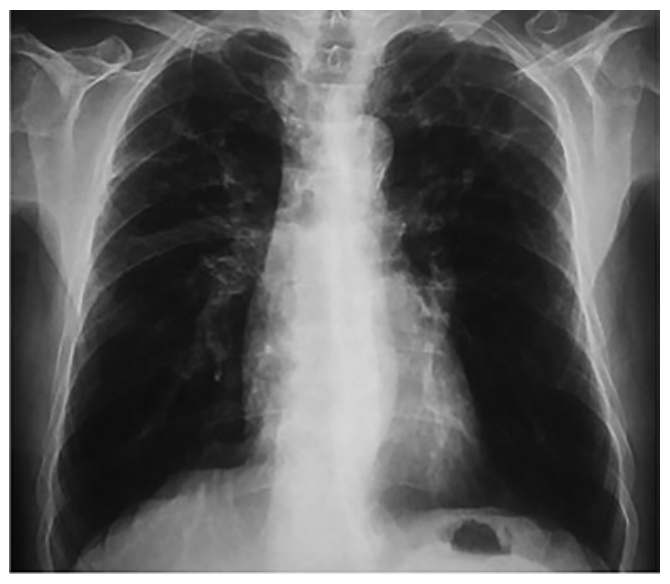

Figure 1 Chest radiograph showing diffuse, small rounded opacities, predominantly in the upper lung lobes and bilateral enlargement of hilar lymph nodes.
Cite as: Tomos I, Tsagkari E, Kefalidou E, et al. Bilateral nodular opacities and hilar node enlargement in a 73-year-old man with cough. Breathe 2018; 14: e55-e58. 


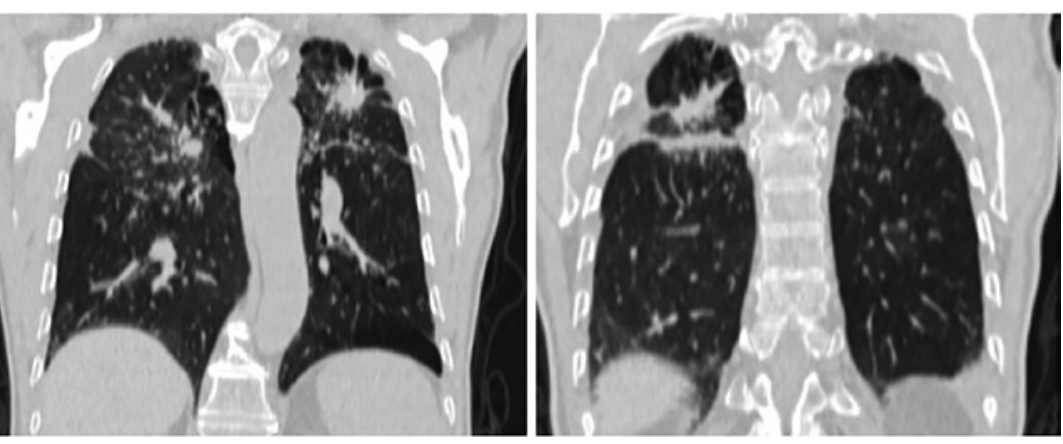

Figure 2 Chest CT revealing the presence of bilateral upper lung nodules with a diameter $>1 \mathrm{~cm}$, with surrounding small nodules and parenchymal distortion.
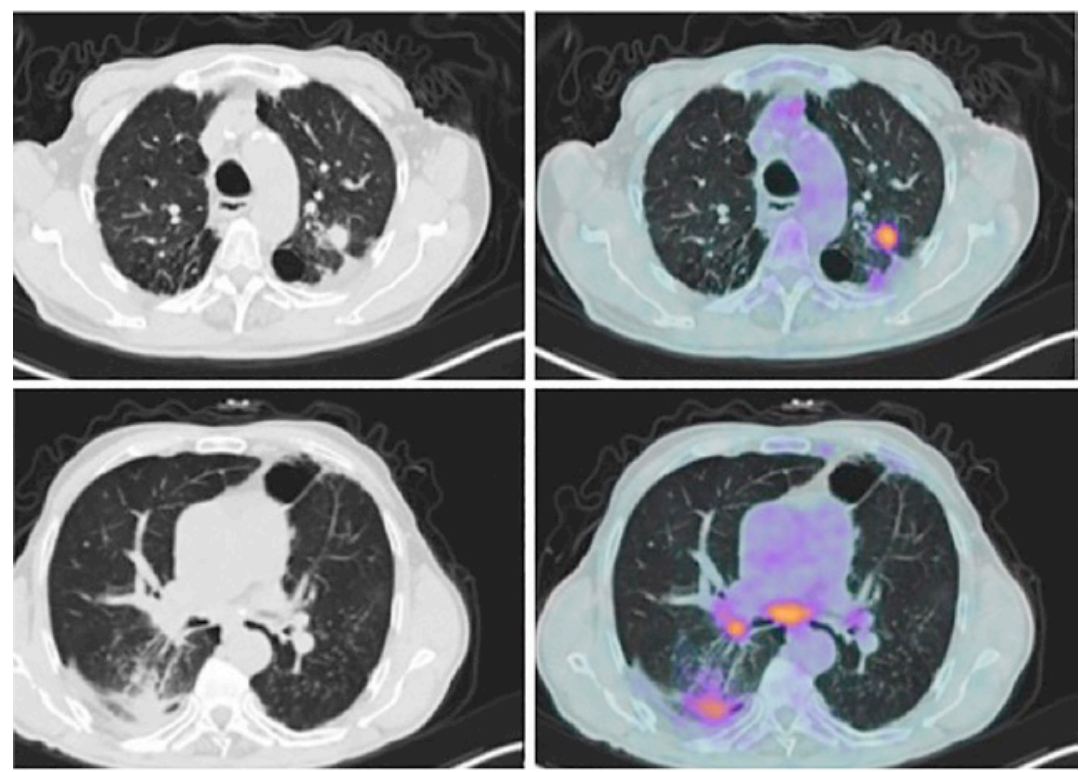

Figure $3{ }^{18}$ F-FDG PET demonstrating hypermetabolic lesions with increased uptake in both hilar lymph nodes and the nodular lesions of the upper lobes.

\section{Answer 1}

Chest computed tomography (CT) was performed to reveal the exact radiological features of the disease.

Chest CT confirmed the presence of bilateral upper lung nodules with a diameter $>1 \mathrm{~cm}$, with adjoining smaller nodules, parenchymal distortion and bilateral enlarged hilar lymph nodes (figure 2). ${ }^{18}$ F-2-fluoro-2-deoxy-D-glucose positron emission tomography $\left({ }^{18} \mathrm{~F}-\mathrm{FDG} \mathrm{PET}\right)$ was performed revealing hypermetabolic lesions with an increased uptake in both hilar lymph nodes and in the nodular lesions of the upper lobes (figure 3).

\section{Task 2}

What could be the differential diagnosis in light of the CT findings?

\section{Answer 2}

The differential diagnosis includes sarcoidosis, lung cancer, progressive massive fibrosis, pneumoconiosis, tuberculosis and histoplasmosis.

Bronchoscopy with transbronchial needle aspiration of the mediastinal lymph nodes was performed. There was no endobronchial lesion. Microbiological examination yielded negative results for common pathogens, Mycobacterium tuberculosis and fungi, while cytological examinations were negative for malignancy and non-compatible for sarcoidosis. His Mantoux test was negative $(0 \mathrm{~mm})$. Spirometry revealed an obstructive pattern with decreased forced expiratory volume in $1 \mathrm{~s}$ (FEV1) ( $55 \%$ predicted) and a $\mathrm{FEV}_{1} /$ forced vital capacity ratio of 0.50 , which was compatible with chronic obstructive pulmonary disease (COPD). A CT-guided biopsy was, therefore, performed to the nodular lesion in the right upper lobe, which revealed fibrosis and inflammatory cells without evidence of malignancy.

Task 3

What is the preliminary diagnosis?

Task 4

Which pathophysiological entities are associated with exposure to respirable silicon dioxide?

Task 5

What is required for the diagnosis of silicosis to be established? 
Answer 3

Progressive massive fibrosis (PMF).

\section{Answer 4}

Diffuse interstitial lung disease (simple chronic silicosis), tuberculosis, lung cancer, PMF and autoimmune diseases.

\section{Answer 5}

A correlated history of exposure to silica, compatible radiographic findings and exclusion of other diseases that may cause similar abnormalities.

The patient was treated with bronchodilators for COPD, and remains under regular medical follow-up with chest radiographs and spirometry. Influenza and pneumococcal vaccination was also suggested.

\section{Discussion}

Silicosis is a fibronodular lung disease caused by the inhalation of crystalline silicon dioxide [1]. Despite the established preventive measures, silicosis remains one of the most frequent occupational diseases worldwide [2]. Especially in developing countries, where industrial hygiene measures have not yet achieved the desired safe levels of mineral dust in the workplace, silicosis constitutes a major public health issue. Conversely, in the developed world, despite a steady decline thanks to the established protective measures (dust control and respirator use), silicosis still constitutes an occupational hazard.

The element silicon is one of the most abundant in nature, comprising $25 \%$ of the earth's crust. It occurs in crystalline and amorphous forms [3]. Common types of free crystalline forms include quartz, tridymite and cristobilite, while less commonly encountered forms are keatite, coesite and stishovite. Rocks, such as sandstone and granite, are formed by quartz in varying concentrations. Occupational exposure to respirable particles of silica (0.2-10 $\mu \mathrm{m}$ aerodynamic diameter) occurs in diverse occupations and industries, including those where rocks and stones are drilled (mining, quarries) or broken down to dust (sculpting, rock cutting) [1].

The diagnosis of silicosis requires three criteria: 1) history of exposure to silica, 2) compatible radiological features and 3 ) exclusion of other diseases with a similar presentation [1]. Symptoms are not specific and resemble those of other chronic respiratory diseases, they include cough and progressive shortness of breath on exertion [1]. Thus, occupational history with exposure is the key factor for clinicians to suspect the disease.
Four clinical patterns have been described in the context of silicosis. Chronic simple silicosis represents the most common form, presenting after prolonged exposure to respirable crystalline free silica, usually after 10 years or more [1, 3]. Patients often present with an incidentally found abnormal radiograph with prominent hilar lymph nodes and calcification or nodules of $<1 \mathrm{~cm}$ in diameter [1]. In $5 \%$ of cases, a distinctive peripheral calcification of the hilar lymph nodes, known as eggshell calcification may be seen [1]. Another pattern is that of PMF, in which nodules are $>1 \mathrm{~cm}$ in diameter and over time progressively increase in size. As these large fibrotic masses contract, associated emphysema develops in the surrounding tissues, while the destruction of the pulmonary capillary network produces increased pulmonary vascular resistance and eventually pulmonary hypertension and cor pulmonale. Accelerated silicosis shares the same features as the classic chronic silicosis form except that the time from initial exposure to silica to presentation of radiographic lesions is much shorter, usually within a 2-5-year period following intense exposure [1]. Finally, acute silicosis presenting as silicoproteinosis is a rare consequence of exposure to high concentrations of silica. Acute silicosis presents with rapidly progressive dyspnoea, respiratory insufficiency and constitutional symptoms, such as fever, fatigue and weight loss [1, 4]. The differential diagnosis includes various diseases that may present with similar features to silicosis, examples include: sarcoidosis, histoplasmosis, chronic fungal infections, tuberculosis, pneumoconiosis, malignancy and idiopathic pulmonary fibrosis [1]. Imaging (mainly chest radiography) constitutes the principal tool of diagnosis. There is limited data concerning the role of ${ }^{18} \mathrm{~F}$-FDG PET scans in the differentiation of potential PMF lesions or PMF-associated lung cancer. Despite its well-known usefulness in monitoring pulmonary nodules, the ${ }^{18} \mathrm{~F}$-FDG PET scan seems to be of limited utility in the evaluation of malignancy in the context of PMF due to its high false-positive rate [5, 6]. Spirometry may be normal, although both obstructive and restrictive patterns are reported in silicosis. However, diffusing capacity of the lung for carbon monoxide seems to be the most sensitive marker in the early stages of the disease [7].

Furthermore, exposure to silica has been associated with a variety of diseases. It is well known that the risk of tuberculosis is increased with the severity of silicosis [8]. Moreover, a number of studies have demonstrated an associated risk for developing COPD, lung cancer and autoimmune diseases, including scleroderma and rheumatoid arthritis, with silica exposure [9-11].

With regards to the pathophysiology of the disease, inhalation of crystalline silica dust leads to deposits in the distal airways. There, silica particles interact with the alveolar macrophages [12]. Various in vitro and animal model experiments 
have demonstrated the activation of the nucleotidebinding domain, leucine-rich repeat protein NALP3, which combines with the intracellular adapter protein ASC activating pro-caspase 1 . The result of this signalling pathway is the production of proinflammatory interleukin-1 $\beta$, which plays a critical role in subsequent inflammation and fibrosis $[13,14]$.

Currently there is no proven effective therapy for silicosis [1]. Whole lung lavage and corticosteroids have been tried in order to slow disease progression; however, there is no evidence of any long-term benefit with their use [15]. General supportive care is the best that can be offered to these patients (smoking cessation, influenza and pneumococcal vaccination, bronchodilator medication and a high level of vigilance for tuberculosis and for malignancy). Regarding prevention: air quality monitoring, enforced use of protective measures (respirators and clothing) and workplace safety with austere exposure control based on national regulatory strategies represent the most important preventative approaches to reduce the risk of exposure and incidence of silicosis [16].

\section{Conflict of interest}

None declared.

\section{References}

1. Leung CC, Yu IT, Chen W. Silicosis. Lancet 2012; 379: 2008-2018.

2. WHO. The Global Occupational Health Network newsletter: elimination of silicosis. Issue No. 12 - 2007. www.who.int/ occupational_health/publications/newsletter/gohnet12e.pdf Date last updated: 2007.

3. National Institute for Occupational Safety and Health. Health effects of occupational exposure to respirable crystalline silica. Cincinnati, Department of Health and Human Services, 2002

4. Seaton A, Legge JS, Henderson J, et al. Accelerated silicosis in Scottish stonemasons. Lancet 1991; 337: 341-344.

5. Reichert M, Bensadoun ES. PET imaging in patients with coal workers pneumoconiosis and suspected malignancy. J Thorac Oncol 2009; 4: 649-651.

6. Chung SY, Lee JH, Kim TH, et al. ${ }^{18} \mathrm{~F}$-FDG PET imaging of progressive massive fibrosis. Ann Nucl Med 2010; 24: 21-27.

7. Lopes AJ, Mogami R, Capone D, et al. High-resolution computed tomography in silicosis: correlation with chest radiography and pulmonary function tests. J Bras Pneumol 2008; 34: 264-272.

8. Cowie RL. The epidemiology of tuberculosis in gold miners with silicosis. Am J Respir Crit Care Med 1994; 150 1460-1462.
9. Rushton L. Chronic obstructive pulmonary disease and occupational exposure to silica. Rev Environ Health 2007; 22: 255-272.

10. Pelucchi C, Pira E, Piolatto G, et al. Occupational silica exposure and lung cancer risk: a review of epidemiological studies 1996-2005. Ann Oncol 2006; 17: 1039-1050.

11. Makol A, Reilly MJ, Rosenman KD. Prevalence of connective tissue disease in silicosis (1985-2006) - a report from the state of Michigan surveillance system for silicosis. Am J Ind Med 2011; 54: 255-262.

12. Huaux F. New developments in the understanding of immunology in silicosis. Curr Opin Allergy Clin Immunol 2007; 7: 168-173.

13. Hornung V, Bauernfeind F, Halle A, et al. Silica crystals and aluminum salts activate the NALP3 inflammasome through phagosomal destabilization. Nat Immunol 2008; 9: 847-856.

14. Cassel SL, Eisenbarth SC, lyer SS, et al. The Nalp3 inflammasome is essential for the development of silicosis. Proc Natl Acad Sci USA 2008; 105: 9035-9040.

15. Wilt JL, Banks DE, Weissman DN, et al. Reduction of lung dust burden in pneumoconiosis by whole-lung lavage. J Occup Environ Med 1996; 38: 619-624.

16. Kromhout $H$. Design of measurement strategies for workplace exposures. Occup Environ Med 2002; 59: 349-354. 